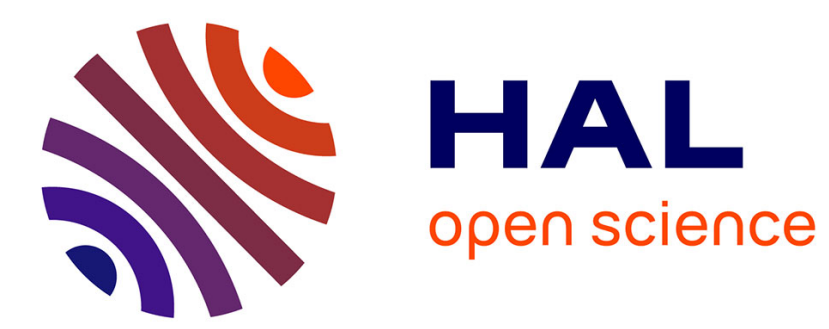

\title{
Optic-Flow Based Car-Like Robot Operating in a 5-Decade Light Level Range
}

Stefano Mafrica, Alain Servel, Franck Ruffier

\section{To cite this version:}

Stefano Mafrica, Alain Servel, Franck Ruffier. Optic-Flow Based Car-Like Robot Operating in a 5Decade Light Level Range. IEEE International Conference on Robotics and Automation (ICRA 2016), IEEE, May 2016, Stockholm, Sweden. hal-01322837

\section{HAL Id: hal-01322837 https://hal.science/hal-01322837}

Submitted on 2 Jun 2016

HAL is a multi-disciplinary open access archive for the deposit and dissemination of scientific research documents, whether they are published or not. The documents may come from teaching and research institutions in France or abroad, or from public or private research centers.
L'archive ouverte pluridisciplinaire HAL, est destinée au dépôt et à la diffusion de documents scientifiques de niveau recherche, publiés ou non, émanant des établissements d'enseignement et de recherche français ou étrangers, des laboratoires publics ou privés. 


\title{
Optic-Flow Based Car-Like Robot Operating in a 5-Decade Light Level Range*
}

\author{
Stefano Mafrica, ${ }^{1,2}$ Alain Servel ${ }^{2}$ and Franck Ruffier ${ }^{1}$
}

\begin{abstract}
In this paper, we present (i) a novel bio-inspired 1-D OF sensor which is robust to high-dynamic-range lighting conditions and independent of the visual patterns encountered, and (ii) a low-cost car-like robot called BioCarBot, which estimates its velocity and steering angle by means of an Extended Kalman Filer (EKF) using only the OF measurements delivered by two downward-facing sensors of this kind. Indoor experiments were carried out, in which the robot was driven in the closed-loop mode, using a proportional integral (PI) controller based on the velocity and steering angle estimates. The results presented here show that our novel OF sensor can deliver a wide range of high-frequency $(333 \mathrm{~Hz})$ OF measurements (from 1 to $10 \frac{\mathrm{rad}}{\mathrm{s}}$ ) with a relatively high resolution (up to $0.05 \frac{\mathrm{rad}}{\mathrm{s}}$ ) in a 5-decade high-dynamic range of light levels. Neither the refresh rate nor the resolution of the $O F$ sensors presented here depended on either the visual patterns or the lighting conditions, and could be theoretically set at whatever value required.
\end{abstract}

\section{INTRODUCTION}

The sensing techniques traditionally used for motion estimation in the fields of mobile robotics and automobile, such as wheel odometry, inertial measurement units (IMUs) and global navigation satellite systems (GNSSs), such as standard or differential GPSs, often suffer from drift, low resolution, high noise levels and limited applicability [1], [2].

Several approaches based on visual odometry have therefore been recently developed, using standard cameras [3], [4] and optical-mouse sensors [5], [6] as well as custom-made optic flow (OF) sensors [7], [8]. In particular, applications using downward-facing visual sensors have been widely used to estimate the velocity and the distance traveled, since the visual patterns and light conditions encountered are more uniform, and they make it possible to use visual sensors such as optical mice.

On the one hand, most of the solutions based on standard cameras, such as those presented in [3], [4], have disadvantages such as failing to cope with high-dynamic-range lighting conditions and their low-frequency, high computationalcost image processing, which means that only a small range of low velocity measurements can often be obtained. These

*This work was supported by CNRS Institutes (Life Science; Information and Engineering Science and Technology), Aix-Marseille University, ANRT (Association Nationale de la Recherche et de la Technologie), PSA Peugeot Citroën via the OpenLab agreement entitled "Automotive Motion Lab".

${ }^{1}$ Stefano Mafrica and Franck Ruffier are with AixMarseille Université, CNRS, ISM UMR 7287, 13288 Marseille, France stefano.mafrica@univ-amu.fr; franck.ruffiereuniv-amu. fr

${ }^{2}$ Stefano Mafrica and Alain Servel are with PSA Peugeot Citroën, Route de Gisy, 78140 VélizyVillacoublay, France stefano.mafrica@mpsa.com; alain.servel@mpsa.com two issues can be improved by using high-dynamic-range (HDR), high-frequency cameras, but these solutions would require even larger computational resources and would be too expensive for most robotic and automotive applications.

On the other hand, solutions based on optical mice, such as those presented in [5], [6], are very cheap and deliver highfrequency measurements but have the main disadvantage of having to operate very close to the ground to be able to work properly, and are therefore unsuitable for uneven-terrain applications. In addition, these sensors are usually highly sensitive to the lighting conditions and like standard cameras, deliver measurements in a rather small velocity range.

Solutions based on custom laser or LED-lighted OF sensors, such as those presented in [7], [8], have been developed in order to reduce the sensitivity to height and improve the performances while traveling over terrains of various kinds, but no tests were run by these authors under various lighting conditions and under the robot's normal driving conditions.

Several versions of Local Motion Sensors (LMSs) providing alternative solutions to standard visual sensors have been developed at our laboratory [9]-[12] to obtain 1-D OF measurements in various velocity and luminosity ranges, using methods based on findings obtained on the fly's visual system [13].

In this paper, we present (i) a novel bio-inspired OF sensor giving measurements which are robust to high-dynamicrange lighting conditions and do not depend on the visual patterns encountered, and (ii) a low-cost car-like robot called BioCarBot, which estimates its velocity and steering angle by means of an Extended Kalman Filer (EKF) using only the OF measurements delivered by two downward-facing sensors of this kind (see Fig. 11). To test the performances of the OF sensors and the method presented here for estimating the velocity and the steering angle, some indoor experiments were carried out, which consisted in driving the robot on circular trajectory in the closed-loop mode using a proportional integral (PI) controller based on the velocity and steering angle estimates obtained.

The results presented here show that our novel OF sensor is robust to high-dynamic-range lighting conditions (in a 5decade range with sharp changes of up to 2 decades within $0.5 \mathrm{~s}$ ), delivering a wide range of high-frequency $(333 \mathrm{~Hz})$ OF measurements (from 1 to $10 \frac{\mathrm{rad}}{\mathrm{s}}$, i.e. 50 to $550 \frac{\mathrm{o}}{\mathrm{s}}$ ) with a relatively high resolution (up to $0.05 \frac{\mathrm{rad}}{\mathrm{s}}$ ). It is also worth noting that (i) the refresh frequency of the LMS was constant and did not depend on either the band-pass filter or the visual patterns encountered, and (ii) the resolution was nearly constant and could be theoretically set at any value. 


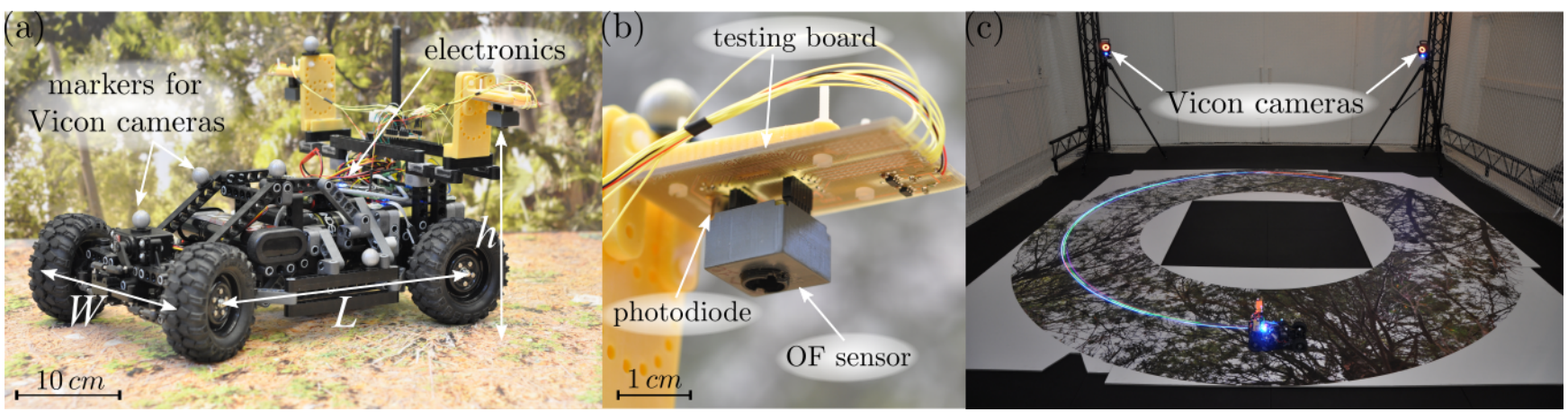

Fig. 1. (a) Picture of the BioCarBot robot. (b) Picture of one of the 2 OF sensors used on the robot. (c) Picture of the indoor testing environment equipped with a Vicon motion-capture system.

In Sec. III we will first introduce the principles underlying an elementary LMS before describing the method and the hardware used to construct our novel OF sensor. In Sec. [III. we will present the BioCarBot robot, the mathematical modeling used to estimate the robot's velocity and steering angle, and the estimation and control scheme implemented on the robot. In Sec. IV] we will present and discuss the results obtained in the indoor experiments performed. Some conclusions will be reached in the last section.

\section{New Implementation of the Local Motion SENSOR}

\section{A. Principles Underlying a LMS}

A defocused lens placed in front of 2 photoreceptors determines the interreceptor angle $\Delta \varphi$ between the 2 photoreceptors' axes and gives them a Gaussian angular sensitivity with an acceptance angle $\Delta \rho$ (Fig. 2(a)), on similar lines to what occurs in many insects' eyes [13]. A visual contrast moving in front of the LMS will induce a time lag $\tau$ between the photoreceptors' output signals (Fig. 2(b)). After measuring this time lag, the optic flow can be computed as follows [9]:

$$
\omega(t)= \pm \frac{\Delta \varphi}{\tau(t)},
$$

where the sign depends on the sensor's axis orientation and on which of the two signals is delayed.

The acceptance angle $\Delta \rho$, namely the full width at halfheight of the Gaussian curve, determines the cut-off frequency of the low-pass spatial filter.

\section{B. New Method for Computing the OF}

To compute the OF measurement $\omega$, the time lag $\tau$ between 2 neighboring-pixels' output signals was estimated using a cross-correlation method inspired by the ReichardtHassenstein model [14]. First the 2 pixels' output signals $\left(V_{P h_{1}}, V_{P h_{2}}\right)$ were sampled and band-pass filtered $\left(V_{P h_{1}}^{\prime}, V_{P h_{2}}^{\prime}\right)$, and the following pseudo-algorithm was then applied (Fig. 2(c),(d)):

1) delay one of the 2 filtered signals by the time $\tau_{i}$;

2) compute the Pearson correlation coefficients between the delayed (e.g. $V_{P h_{1}}^{\prime}\left(t-\tau_{i}\right)$ ) and non-delayed signal (e.g. $\left.V_{P h_{2}}^{\prime}(t)\right)$ in a fixed time window $w_{P h}$;
3) repeat step 1 and 2 for every $\tau_{i}(i=1, \ldots, n)$ in a fixed time window $w_{\tau}$

4) set $\tau$ at a value equal to the time lag $\tau_{k}$ giving the maximum cross-correlation coefficient, as long as this maximum is greater than a fixed value $\rho_{t h r}$;

5) compute $\omega$ using the equation (1) (or set it at $\mathrm{NaN}$ if the maximum cross-correlation coefficient is less then $\left.\rho_{\text {thr }}\right)$.

The threshold value of the cross-correlation coefficients $\left(\varrho_{t h r}\right)$ directly determines the reliability and the robustness of the OF measurements: the higher $\varrho_{t h r}$ is, the more reliable and robust the measurements will be, but in the presence of noise, the lower the refresh rate will be.

The time window of the signals $\left(w_{P h}\right)$ determines the bandwidth of the OF measurements, as the time lag $\tau$ must be nearly constant during this time in order to obtain a strong correlation, whereas the number of samples $(n)$ in the time window determines the reliability of the correlation coefficients: the smaller $w_{P h}$ is, the larger the bandwidth will be, and the higher $n$ is, the higher the reliability will be.

The time window of the time lags $\left(w_{\tau}\right)$ determines the range of the OF measurements $\left(\omega_{\min }=\frac{\Delta \varphi}{\tau_{m}}, \omega_{\max }=\frac{\Delta \varphi}{\tau_{0}}\right)$, whereas the difference between two consecutive time lags $\left(\Delta \tau=\tau_{i+1}-\tau_{i}\right)$ determines the resolution of the OF measurements $\left(\Delta \omega=\omega_{i+1}-\omega_{i}\right)$. In order to obtain a constant resolution $\Delta \omega$, the time lags were chosen as follows:

$$
\tau_{i}=\frac{\Delta \varphi}{\left|\omega_{i}^{*}\right|},
$$

where $\omega_{i}^{*}$ are the desired OF measurements, which are linearly separated by the required resolution $\Delta \omega^{*}$. The signals can then be delayed by a time $\tau_{i}$ elapsing between two sampling steps, by linearly interpolating the signals sampled.

\section{Hardware Implementation}

In this study, we used the auto-adaptive silicon retina presented in [15] soldered onto a tiny printed circuit board (PCB) with an optical lens casing mounted onto it (Fig. 3).

The $\mathrm{M}^{2} \mathrm{APix}$ pixel, which stands for Michaelis-Menten Auto-Adaptive Pixel, can auto-adapt in a 7-decade range and responds appropriately to small contrasts, such as $\pm 2 \%$, as well as large changes in light, such as \pm 3 decades [15]. 


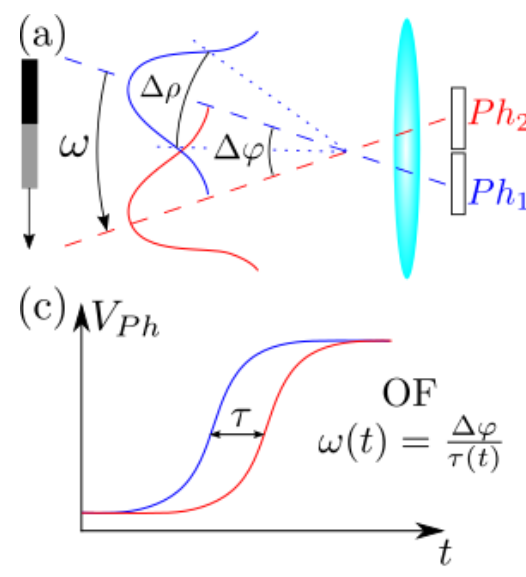

(b)
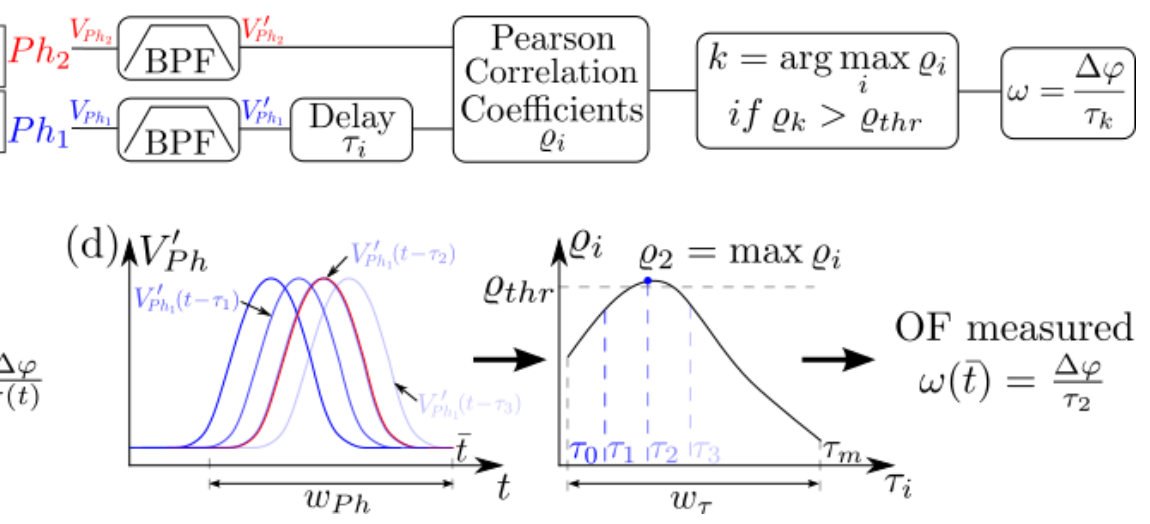

Fig. 2. (a),(c) Principles underlying a 2-pixel LMS. The optic flow produced by a contrast moving in front of the LMS can be computed taking $\omega(t)=\frac{\Delta \varphi}{\tau(t)}$, where $\Delta \varphi$ is the interreceptor angle and $\tau$ is the time lag between the two photoreceptors' output signals [9]. (b),(d) Block diagram of the novel OF algorithm inspired by the Reichardt-Hassenstein model [14]. After band-pass filtering the photoreceptors' outputs, one of the two signals is delayed by a time $\tau_{i}$ in a fixed time window and the Pearson cross-correlation coefficient $\varrho_{i}$ between the delayed and non-delayed signals is computed. The $\mathrm{OF}$ is then computed taking $\omega= \pm \frac{\Delta \varphi}{\tau_{k}}$, where $\tau_{k}$ is the time lag giving the maximum cross-correlation, as long as this maximum is greater than $\varrho_{t h r}$ (which is usually set at 0.99 ). The sign of $\omega$ depends on the sensor's axis orientation and on which signal is delayed.
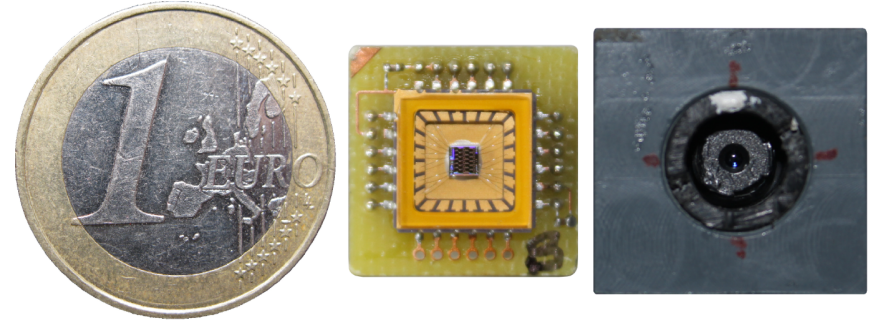

Fig. 3. Picture of the auto-adaptive silicon retina comprising the 12 $\mathrm{M}^{2} \mathrm{APix}$ pixels [15] soldered onto a tiny PCB and the optical lens casing mounted onto it.

In the chip used here, the analog low-pass filter had a cutoff frequency of $100 \mathrm{~Hz}$ (instead of $300 \mathrm{~Hz}$ used in [15]), giving a minimum sampling frequency of $200 \mathrm{~Hz}$ in order to prevent the occurrence of aliasing.

The optical lens used here was taken from a RaspberryPi camera (focal length: $2 \mathrm{~mm}$ ), while the lens casing was custom made using a 3D printer to precisely adjust the distance between the plane of the lens and that of the pixels during the calibration phase. The interreceptor angle $\Delta \varphi$ and the acceptance angle $\Delta \rho$ were measured at $\Delta \varphi \approx \Delta \rho \approx$ $3.6^{\circ}$, as occurs in some diurnal insects [16], using a similar method to that presented in [10].

\section{BioCarBot: a Bio-Inspired Visually-Guided CAR-LIKE ROBOT}

\section{A. The Robot}

Figure 1 shows a picture of: the BioCarBot robot, one of the 2 LMSs used and the indoor testing environment equipped with a Vicon motion-capture system.

The main structure of the BioCarBot robot was based on the low-cost 2WD Racecar Kit provided by Minds-I Robotics. The kit was composed of a 1/10th-scale car body $(419 \times 203 \times 114 \mathrm{~mm})$, one Hitec HS-311 standard servo coupled to a steering hub, one 5000-rpm DC motor connected to a 300-A electronic speed controller (ESC), one 7.2Volt 3000-mAh Ni-Cd rechargable battery, one mechanical slip differential and four $90 \mathrm{~mm}$-diameter crawler wheels. We replaced the 23500-rpm DC motor and the four $66 \mathrm{~mm}$ diameter wheels included in the original kit in order to provide the robot with a higher control resolution at low velocity and the ability to drive over uneven terrains.

The embedded electronics include one Nanowii board (Flyduino) featuring a ATmega32u4 16-MHz CPU microcontroller (Atmel) and a MPU-6050 inertial measurement unit (IMU) comprising a 3-axis gyroscope and a 3-axis accelerometer (InvenSense) and one Overo IronSTORM computer-on-module (COM) (Gumstix) featuring a $1-\mathrm{GHz}$ CPU DM3730 processor (Texas Instruments) comprising an ARM Cortex-A8 architecture and a C64x digital signal processor (DSP).

Thanks to the modularity of the robot's structure, 2 identical LMSs (Fig. 3 b)) were attached to the robot's frame on both sides of its body, aligned with the rear wheel axis (Fig. 11a)). To facilitate the sensors' installation, we used the same testing board which was used in the study presented in [15] to connect the LMSs to the Nanowii board (Fig. 1(b)). An OSRAM BPX65 photodiode connected to an analog amplifier circuit was also included on the testing board next to the LMS in order to measure the effective illuminace of the scene scanned by the sensor.

To obtain the ground-truth values, the 3-D robot's pose $\left([X Y Z \alpha \beta \gamma]^{T}\right)$ was measured by means of a Vicon motion-capture system [17] which detected the position of the infrared markers attached to the robot's frame (see Fig. $1(a),(c))$.

\section{B. Modeling}

As the robot's velocity was relatively low and the robot did not have any suspension system, we focused here on the 2-D 
kinematic model for a car-like robot moving on a flat surface. Figure 4 shows the kinematic diagram of the BioCarBot with the 2 LMSs installed on both sides, as depicted in Fig. 1. a).

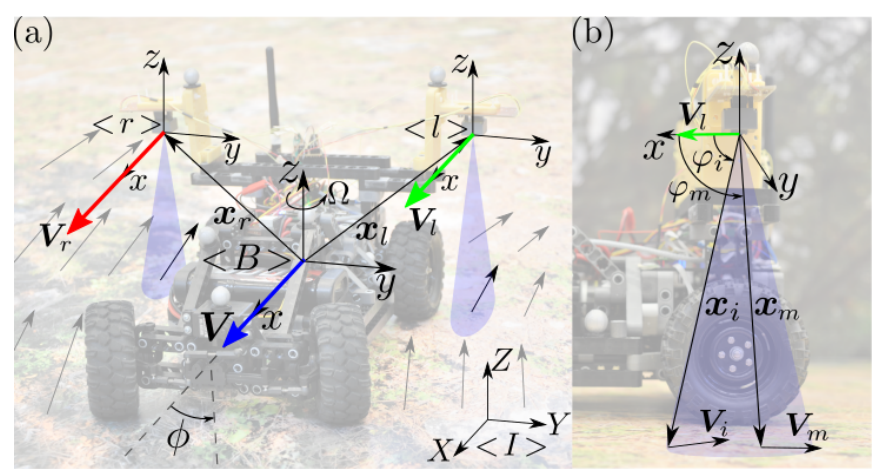

Fig. 4. (a) Kinematic diagram of the robot moving on a flat surface. (b) Kinematic diagram of one OF sensor.

Let us take the inertial frame $\langle I>$ having the $x$ and $y$ axes lying on the local ground plane, with the robot's body frame $<B>$ placed in the middle of the rear wheels' axis. Two LMSs were placed at $\boldsymbol{x}_{l}=\left[x_{l} y_{l} z_{l}\right]^{T}$ and $\boldsymbol{x}_{r}=\left[\begin{array}{lll}x_{r} & y_{r} & z_{r}\end{array}\right]^{T}$ with respect to $<B>\left(x_{l}=x_{r}=0 \mathrm{~mm}\right.$, $\left.y_{l}=-y_{r}=140 \mathrm{~mm}, z_{l}=z_{r}=125 \mathrm{~mm}\right)$, respectively, facing downwards at a height of $h_{l}, h_{r}$, respectively, from the ground $\left(h_{l}=h_{r}=h=175 \mathrm{~mm}\right.$ ) (Fig. 1 a) 4(a)). As the sensors' frames $\langle l\rangle,\langle r\rangle$ were taken to be parallel to the body frame $\langle B\rangle$, we can consider all the position and velocity vectors projected onto $\langle B\rangle$.

The OF produced by the visual motion of the ground with respect to the sensor can be measured using the method presented in section II-B based on the no-skidding hypothesis, which guarantees that $V_{x}>>V_{y}$ with any velocity vector $\boldsymbol{V}=\left[V_{x} V_{y} 0\right]^{T}$ located near the line passing through the two rear wheel/ground contact points. Therefore, the OF measured between the $i-1$-th and $i$-th pixels of each LMS can be written as follows:

$$
\omega_{i}=-\frac{V_{i_{x}} \sin ^{2} \varphi_{i}}{h},
$$

where $\boldsymbol{V}_{i}=\left[V_{i_{x}} V_{i_{y}} 0\right]^{T}$ is the velocity vector of the intersection point $\boldsymbol{x}_{i}=\left[x_{i} y_{i} z_{i}\right]^{T}\left(y_{i} \approx 0, z_{i} \approx-h\right)$ between the $i$-th pixel axis and the ground plane (Fig. 4(b)).

Since the two LMSs are fixed to the robot so that the rows of pixels are aligned with the $x$-axis of $\langle B\rangle$ (Fig. $4(\mathrm{a})$ ), all the position vectors $\boldsymbol{x}_{i}$ of each LMS have the same $y$ coordinate, namely $y_{l}$ and $y_{r}$ on the left and right sides, respectively. Therefore, according to the Ackermann steering geometry [18], the following equations hold:

$$
\left\{\begin{array}{l}
V_{i_{x}}^{l}=V_{l}=V-y_{l} \Omega \\
V_{i_{x}}^{r}=V_{r}=V-y_{r} \Omega \\
\Omega=\frac{\tan \phi}{L} V
\end{array},\right.
$$

where $L(=255 \mathrm{~mm})$ is the distance between the rear and front wheel axes (Fig. 1 (a)), and $\Omega, V, \phi$ are the robot's angular velocity, longitudinal velocity and steering angle, respectively (Fig. 4(a)).

By substituting the median value of the OF measurements of each LMS $\omega_{m}^{l}, \omega_{m}^{r}$ into (2) and combining equations (2) and (3), we obtain the following equation relating the output $\boldsymbol{\zeta}=\left[\omega_{m}^{l} \omega_{m}^{r}\right]^{T}$ to the state $\boldsymbol{\xi}=[V \phi]^{T}$ :

$$
\boldsymbol{\zeta}=\left[\begin{array}{c}
\omega_{m}^{l} \\
\omega_{m}^{r}
\end{array}\right] \approx\left[\begin{array}{l}
\frac{\left(L-y_{l} \tan \phi\right) V \sin ^{2} \varphi_{m}^{l}}{h L} \\
\frac{\left(L-y_{r} \tan \phi\right) V \sin ^{2} \varphi_{m}^{r}}{h L}
\end{array}\right]=\boldsymbol{h}(\boldsymbol{\xi}),
$$

where $\varphi_{m}^{l}, \varphi_{m}^{r}$ are the orientation of the pixel's axis corresponding to the median OF values $\omega_{m}^{l}, \omega_{m}^{r}$, respectively.

Lastly, the dynamics of $V$ and $\phi$, which mostly depend on the dynamics of the DC motor and the steering servo, respectively, were identified as two independent first-order systems using the Vicon's measurements:

$$
\dot{\boldsymbol{\xi}} \approx A \boldsymbol{\xi}+B \boldsymbol{u}=\boldsymbol{f}(\boldsymbol{\xi}, \boldsymbol{u}) .
$$

where $A=\operatorname{diag}\left(a_{1}, a_{2}\right), B=\operatorname{diag}\left(b_{1}, b_{2}\right)$.

Since the identified time constant describing the dynamics of $V$ when it was decreasing was nearly twice as high as when it was increasing, the values of $A$ and $B$ were identified using only the slower time constants as follows: $a_{1}=-b_{1}=$ $-2.15, a_{2}=-b_{2}=-4.87$.

It is worth noting that the values of $V$ and $\phi$ could be computed algebraically by inverting equation (4), however this estimation would not be accurate enough to perform a closed-loop control on $V, \phi$ due to the noise and the quantization of the OF measurements. We therefore estimated $V, \phi$ by means of an Extended Kalman Filter (EKF) based on equation (5) and (4), as described in the next subsection.

\section{Estimation and Control}

In order to obtain a robust continuous estimation of the robot's longitudinal velocity and steering angle $(V, \phi)$, an Extended Kalman Filter (EKF) based on a discrete approximation for the model presented in (5), (4) was implemented, taking the median value of the OF measurements $\left(\omega_{m}^{l}, \omega_{m}^{r}\right)$ to be actual measurements.

The first-order discrete approximation for the model presented in (5) was taken to be as follows:

$$
\left\{\begin{aligned}
\boldsymbol{\xi}_{k} & =\hat{\boldsymbol{f}}\left(\boldsymbol{\xi}_{k-1}, \boldsymbol{u}_{k-1}, \boldsymbol{w}_{k-1}\right)= \\
& =\left[\boldsymbol{f}\left(\boldsymbol{\xi}_{k-1}, \boldsymbol{u}_{k-1}\right)+\boldsymbol{w}_{k-1}\right] \Delta t+\boldsymbol{\xi}_{k-1} \\
\boldsymbol{\zeta}_{k} & =\hat{\boldsymbol{h}}\left(\boldsymbol{\xi}_{k}, \boldsymbol{\nu}_{k}\right)=\boldsymbol{h}\left(\boldsymbol{\xi}_{k}\right)+\boldsymbol{\nu}_{k}
\end{aligned}\right.
$$

where the index $k$ denotes the $k$-th sampling period (i.e., $t=k \Delta t) ; \boldsymbol{w}, \boldsymbol{\nu}$ denote the model and the measurement noise, respectively, and they are assumed to be independent white noises and to have normal probability distributions, i.e. $p(w) \sim N(0, Q)$ and $p(\nu) \sim N(0, R)$, where $Q=$ $\operatorname{diag}\left(\sigma_{V}^{2}, \sigma_{\phi}^{2}\right), R=\operatorname{diag}\left(\sigma_{l}^{2}, \sigma_{r}^{2}\right)$ are covariance matrices ${ }^{1}$

At each sampling period, the steps involved in the EKF can be summarized as follows:

\footnotetext{
${ }^{1}$ The hypothesis that the components of the model and the measurement noise had normal and uncorrelated distributions was adopted on the basis of what was observed statistically during several experimental tests with trajectories of various kinds (see section IV.
} 


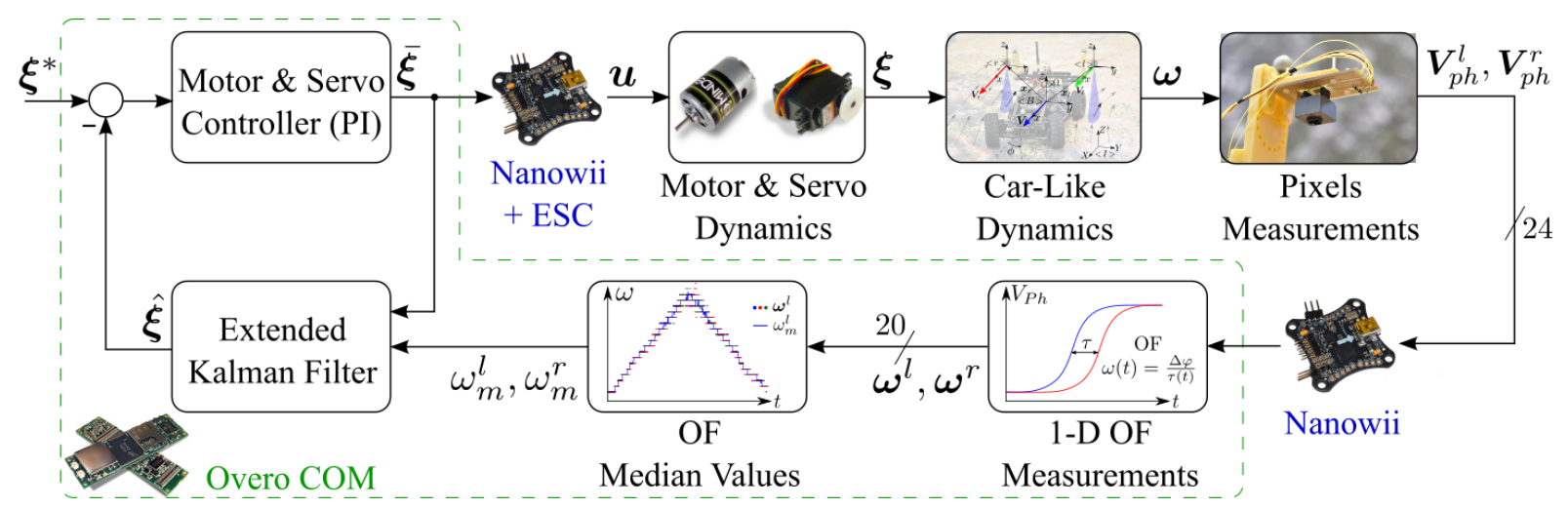

Fig. 5. Block diagram of the estimation and control scheme.

1) $\hat{\boldsymbol{\xi}}_{k}^{-}=\hat{\boldsymbol{f}}\left(\hat{\boldsymbol{\xi}}_{k-1}, \boldsymbol{u}_{k-1}, \mathbf{0}\right)$

2) $P_{k}^{-}=F_{k} P_{k-1} F_{k}^{T}+W_{k} Q W_{k}^{T}$

3) $K_{k}=P_{k}^{-} H_{k}^{T}\left(H_{k} P_{k}^{-} H_{k}^{T}+V_{k} R V_{k}^{T}\right)^{-1}$

4) $\hat{\boldsymbol{\xi}}_{k}=\hat{\boldsymbol{\xi}}_{k}^{-}+K_{k}\left(\boldsymbol{\zeta}_{k}-\hat{\boldsymbol{h}}\left(\hat{\boldsymbol{\xi}}_{k}^{-}, \mathbf{0}\right)\right)$

5) $P_{k}=\left(I-K_{k} H_{k}\right) P_{k}^{-1}$

where $F=\frac{\partial \hat{\boldsymbol{f}}}{\partial \boldsymbol{x}}=\Delta t A+I, H=\frac{\partial \hat{\boldsymbol{h}} 2}{\partial \boldsymbol{x}}, W=\frac{\partial \hat{\boldsymbol{f}}}{\partial \boldsymbol{w}}=\Delta t I$, $V=\frac{\partial \hat{\boldsymbol{h}}}{\partial \boldsymbol{\nu}}=I$ are Jacobian matrices; $P, P^{-}$are the error covariance matrix and its "a priori" estimate, respectively; and $I$ is the identity matrix.

The initial estimate $\hat{\xi}_{0}$ was set at zero, while the initial estimate $P_{0}$ was set at the identity matrix.

As we have no measurements of the real motor and steering control input $\boldsymbol{u}$, we took it to be equal to the output of the controller, i.e. $\boldsymbol{u}_{k-1}=\overline{\boldsymbol{\xi}}_{k-1}$ (see equation (7)).

When there were no measurements on at least one of the robot's sides, i.e. no $\omega_{m}^{l}$ or $\omega_{m}^{r}$, the Kalman gain $K_{k}$ was set at zero, so that it was still possible to have an estimation of $\boldsymbol{\xi}_{k}$ based on the "a priori" prediction $\hat{\boldsymbol{\xi}}_{k}^{-}$. In that case, a timeout was set at $0.5 s$, after which the EKF was reinitialized and the robot was stopped.

The robot's longitudinal velocity and steering angle $(\boldsymbol{\xi}=$ $[V, \phi]^{T}$ ) were controlled in closed loop using the estimated values $\left(\hat{\boldsymbol{\xi}}=[\hat{V}, \hat{\phi}]^{T}\right)$ by means of a proportional and integral (PI) controller, as shown in Fig. 5.

The output from the PI controller can be written as follows:

$$
\overline{\boldsymbol{\xi}}=K_{P}\left(\boldsymbol{\xi}^{*}-\hat{\boldsymbol{\xi}}\right)+K_{I} \int\left(\boldsymbol{\xi}^{*}-\hat{\boldsymbol{\xi}}\right),
$$

where $K_{P}=\operatorname{diag}(0.5,0.4)$ and $K_{I}=\operatorname{diag}(2.5,2)$.

\section{EXPERIMENTAL RESULTS}

Experiments were carried out indoors using various floor patterns and trajectories to test the performances of the OF sensors as well as the estimation and control method presented in the previous section.

$$
H=\frac{1}{h L}\left[\begin{array}{ll}
\left(L-y_{l} \tan \phi\right) \sin ^{2} \varphi_{m}^{l} & -\left(1+\tan ^{2} \phi\right) y_{l} V \sin ^{2} \varphi_{m}^{l} \\
\left(L-y_{r} \tan \phi\right) \sin ^{2} \varphi_{m}^{r} & -\left(1+\tan ^{2} \phi\right) y_{r} V \sin ^{2} \varphi_{m}^{r}
\end{array}\right]
$$

In what follows, the $\mathrm{M}^{2} \mathrm{APix}$ output signals were sampled at $\Delta t=3 \mathrm{~ms}$ by the integrated ADC (see [15] for details), acquired by the Nanowii board via SPI communication and transmitted to the computer-on-module (COM) via serial communication (see section III for details). The OF algorithm presented in Sec. II-B as well as the estimation and control scheme shown in Fig. 5 were run at the same rate on the COM. The Linux-based program running on the COM was entirely generated in the Matlab/Simulink environment using the RT-MaG toolbox [17], a custommade toolbox for real-time applications developed at our laboratory. The host-PC program which conveys the control set points $V^{*}, \phi^{*}$ to the robot $(\mathrm{COM})$ and receives data from the robot (COM) and the Vicon system was also developed in the Matlab/Simulink environment using the RTMaG toolbox.

To implement the OF algorithm, we set (see section III-B for details of the parameters):

- the threshold on the cross-correlation coefficients $\varrho_{t h r}=0.99$

- the number of pixels signals samples $n=70$, giving a signal time window for the cross-correlation computation $w_{P h}=n \Delta t=0.21 \mathrm{~s}$;

- the number of time lags $m=30$, because a larger number would cause saturation of the CPU load since the algorithm's implementation was not optimized.

Depending on the velocity range required, the time lag window $w_{\tau}$ ranged from $6.3 \mathrm{~ms}$ to $63 \mathrm{~ms}$ in order to obtain OF measurements $\omega_{i}$ ranging from $1 \frac{\mathrm{rad}}{\mathrm{s}}$ to $10 \frac{\mathrm{rad}}{\mathrm{s}}$ and a resolution $\Delta \omega^{*}$ required ranging from $0.1 \frac{\mathrm{rad}}{\mathrm{s}}$ to $0.3 \frac{\mathrm{rad}}{\mathrm{s}}$.

The elements of the measurements covariance matrix $(R)$ and the process covariance matrix $(Q)$ of the EKF were set at about $\left(\frac{\Delta \omega^{*}}{2}\right)^{2}$ and $0.01^{2}$, respectively $]^{3}$

The ground-truth values were computed from the Vicon measurements $\left([X Y Z \alpha \beta \gamma]^{T}\right)$ as follows (see section III for details):

$$
\text { - } V_{\text {truth }}=\sqrt{\dot{X}^{2}+\dot{Y}^{2}}
$$

\footnotetext{
${ }^{3}$ The values of $R$ were sometimes adjusted after performing some calibration tests since the standard deviation of the LMS on the right side was always slightly larger than on the left, probably because the pixels' signals were more noisy.
} 


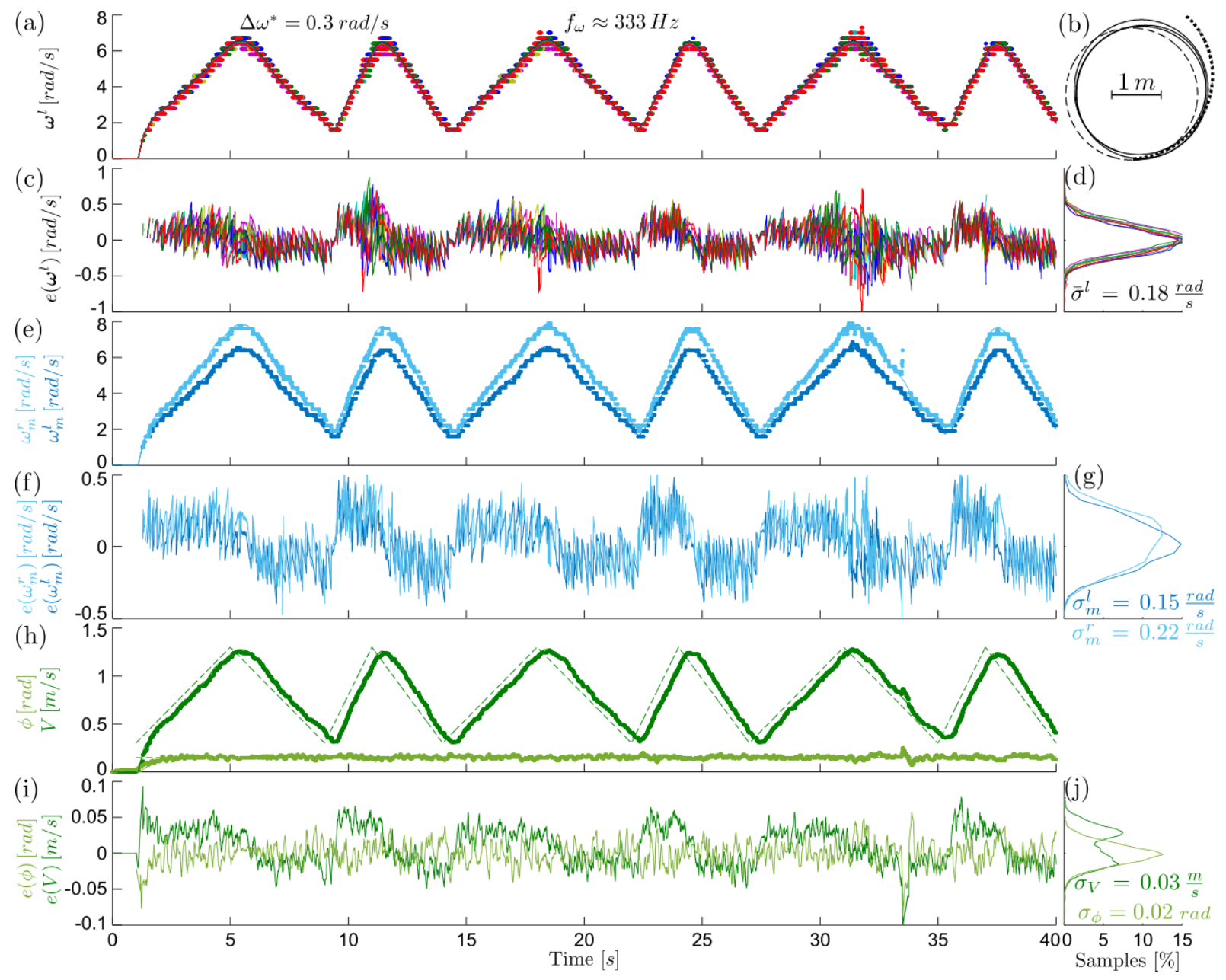

Fig. 6. Results obtained indoors when the robot was driven on the floor shown in Fig. 1 c) on a circular trajectory, keeping a constant steering angle while varying the velocity from 0.3 to $1.3 \frac{\mathrm{m}}{\mathrm{s}}$. (a) Measurements (dots) and ground-truth values (solid lines) of the left OF: each color corresponds to the OF produced between 2 neighboring pixels. (b) Reference (dashed line) and ground-truth robot's trajectory in the closed-loop (solid line) and open-loop (dotted line) mode. (c) Difference between the measurements and the ground-truth values shown in Fig. 6a). (d) Distribution of the errors shown in Fig. 6. c). (e) Median values (dots) and ground-truth values (solid lines) of the OF produced on the left (dark blue) and right (light blue) sides. Some measurements were missing on the right side between 33 and $34 s$ because the robot was driving along the border between the patterned and white areas (see Fig. 11 c)). (f) Difference between the median values and the ground-truth values shown in Fig. 6 e). (g) Distribution of the errors shown in Fig. 66f). (h) EKF estimates (dots), ground-truth (solid lines) and reference values (dashed lines) of the robot's longitudinal velocity (dark green) and steering angle (light green). (i) Difference between the EKF estimates and the ground-truth values shown in Fig. 6h). (j) Distribution of the errors shown in in Fig. G i).

- $\phi_{\text {truth }}=\arctan \left(L_{\frac{\gamma}{V_{\text {truth }}}}\right)$;

- $\omega_{i_{\text {truth }}}^{l}=\frac{\left(y_{l} \dot{\gamma}-V_{\text {truth }}\right) \sin ^{2} \varphi_{i}}{h}$ (same for $\omega_{i_{\text {truth }}}^{r}$ using $\left.y_{r}\right)$.

Figure 6 shows the results obtained indoors when the robot was driven on the floor shown in Fig. 11(c) on a circular trajectory, keeping a constant steering angle while varying the velocity from 0.3 to $1.3 \frac{\mathrm{m}}{\mathrm{s}}$. The OF resolution $\Delta \omega^{*}$ required was set at $0.3 \frac{\mathrm{rad}}{\mathrm{s}}$.

First it should be noted that although the error in the OF measurements increased slightly with the velocity and depended on whether the velocity was increasing or decreasing (Fig. 6(a),(c),(e),(f)), the overall error distribution was nearly Gaussian with a quasi-zero mean (Fig. 6(d), (g)). The average standard deviation of the errors in the $\mathrm{OF}$ measurements was $\bar{\sigma}^{l}=0.18 \frac{\mathrm{rad}}{\mathrm{s}}, \bar{\sigma}^{r}=0.25 \frac{\mathrm{rad}}{\mathrm{s}}$, giving a standard deviation of the error in the OF median values $\sigma_{m}^{l}=0.15 \frac{\mathrm{rad}}{\mathrm{s}}, \sigma_{m}^{r}=0.22 \frac{\mathrm{rad}}{\mathrm{s}}$, which is about half of $\Delta \omega^{*}$, as was to be expected.

Secondly, it is worth noting that the robot adopted the required velocity and steering angle, giving the precision of the estimates $\sigma_{V}=0.03 \frac{\mathrm{m}}{\mathrm{s}}, \sigma_{\phi}=0.02 \mathrm{rad}$ (Fig. 6(h),(i),(j)) and a maximum error in the position of about $30 \mathrm{~cm}$ after 3 laps (solid line in Fig. 6(b)). When the robot was driven in the open-loop mode, however, i.e. without applying the control scheme presented in Fig. 5, it started to drift at a very early stage, going off the carpet after $6 s$ (dotted line in Fig. 6(b)).

Figure 7 gives the results obtained indoors when the robot was driven on the floor shown in Fig. 1.(c) on a circular trajectory, keeping a constant velocity and steering angle under dynamically changing lighting conditions. The OF resolution $\Delta \omega^{*}$ required was set at $0.1 \frac{\mathrm{rad}}{\mathrm{s}}$. 


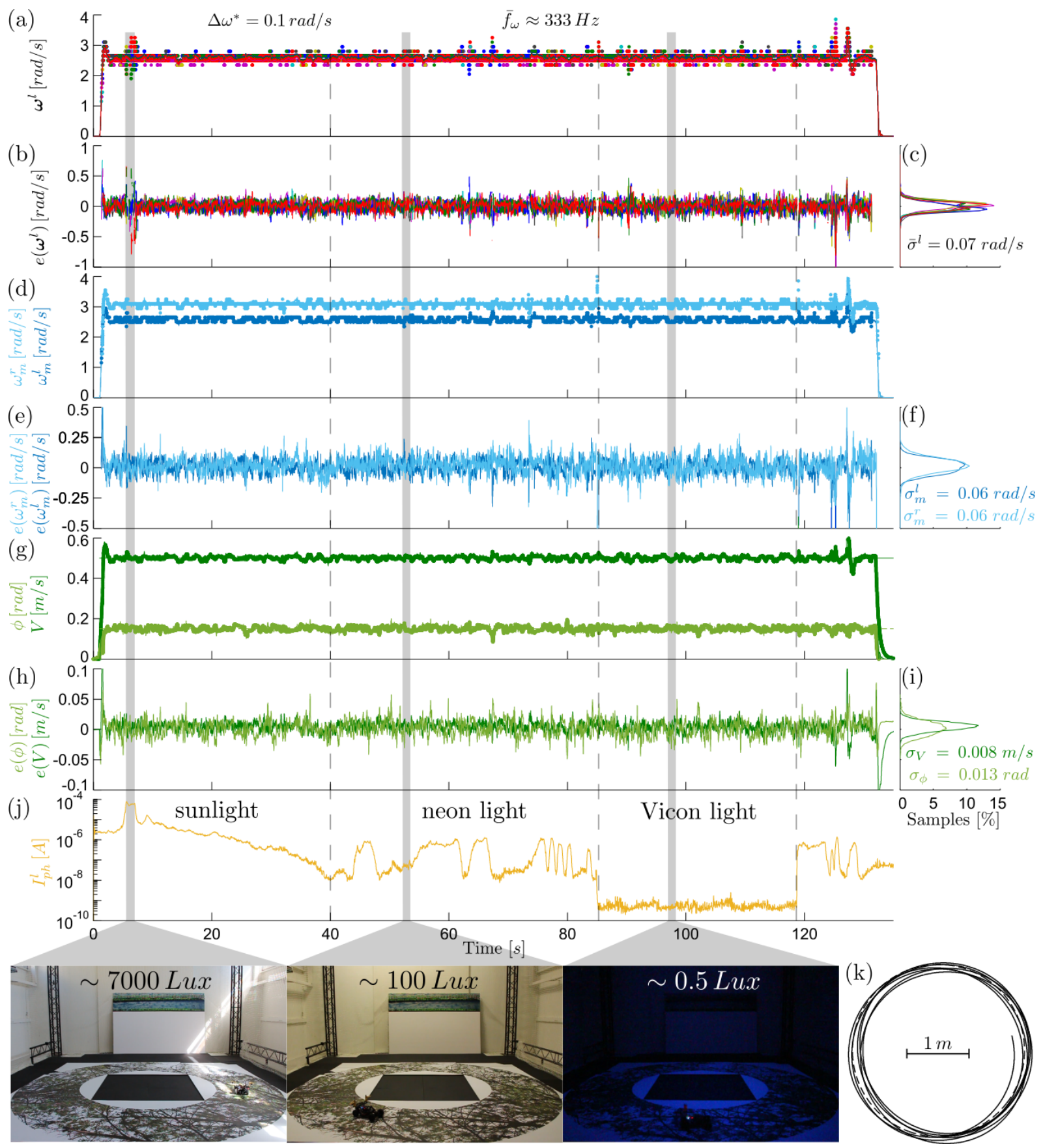

Fig. 7. Results obtained indoors when the robot was driven on the floor shown in Fig. 1 c) on a circular trajectory, keeping a constant velocity and steering angle under dynamically changing lighting conditions. (a) Measurements (dots) and ground-truth values (solid lines) of the left OF: each color corresponds to the OF produced between 2 neighboring pixels. (b) Difference between the measurements and the ground-truth values shown in Fig. 7 a). (c) Distribution of the errors shown in Fig. 7 b). (d) Median values (dots) and ground-truth values (solid lines) of the OF produced on the left (dark blue) and right (light blue) sides. (e) Difference between the median values and the ground-truth values shown in Fig. 7 7 d). (f) Distribution of the errors shown in Fig. $7 \mathrm{le}$ ). (g) EKF estimates (dots), ground-truth (solid lines) and reference values (dashed lines) of the robot's longitudinal velocity (dark green) and steering angle (light green). (h) Difference between the EKF estimates and the ground-truth values shown in Fig. 7) g). (i) Distribution of the errors shown in Fig. 7 h). (j) Photo-current responses of the photodiode placed next to the LMSs on the left side. The current values were computed taking $I_{P h}=I_{\text {dark }}\left(e^{8} 8 V_{\text {out }}-1\right)$, where $I_{\text {dark }}(\approx 0.1 n A)$ is the dark current and $V_{\text {out }}$ is the amplifier's output voltage, and the light values in $L u x$ were estimated using the current-to-illuminance curve given in the photodiode's datasheet. (k) Robot's reference (dashed line) and ground-truth (solid line) trajectories. 
First we varied the incoming sunlight by up to 2 decades, giving a maximum luminosity of about 7000 Lux $\left(I_{p h} \approx\right.$ $7 \times 10^{-5} A$ ), by slowly closing the 8 blinds (from 0 to $40 \mathrm{~s}$ ), and then we varied the artificial lighting by about 2 decades, both slowly and rapidly, by varying the neon ceiling lights and then switching them off (from 40 to $85 \mathrm{~s}$ ). The robot was then driven for a while with only the Vicon cameras' LED lights switched on (from 85 to $119 s$ ), corresponding to a luminosity of about $0.5 \mathrm{Lux}$ (average $I_{p h}$ of about $5 \times$ $10^{-10} A$ ), before switching the neon lights on again (Fig. $7(\mathrm{j})$ ). The light levels tested therefore covered a 5-decade range $\left(I_{p h}\right.$ from about $3 \times 10^{-10}$ to $\left.7 \times 10^{-5} A\right)$.

The OF measurements did not depend on either the average light level or the changes in the light, except in the case of a few very large, sudden changes (e.g. those occurring at $t \approx 5,85,119 \mathrm{~s})$. The overall error distribution was nearly Gaussian with a quasi-zero mean (Fig.7 (b),(c),(e),(f)), giving the average standard deviation of the errors in the OF measurements $\bar{\sigma}^{l}=0.07 \frac{\mathrm{rad}}{\mathrm{s}}, \bar{\sigma}^{r}=0.08 \frac{\mathrm{rad}}{\mathrm{s}}$ and the standard deviation of the error in the OF median values $\sigma_{m}^{l}=0.06 \frac{\mathrm{rad}}{\mathrm{s}}, \sigma_{m}^{r}=0.06 \frac{\mathrm{rad}}{\mathrm{s}}$, which is about half of $\Delta \omega^{*}$, as was to be expected.

The robot adopted the required velocity and steering angle, giving the estimate precision $\sigma_{V}=0.008 \frac{\mathrm{m}}{\mathrm{s}}, \sigma_{\phi}=0.013 \mathrm{rad}$ (Fig. 7(g),(h),(i)) and a maximum error in the position of about $20 \mathrm{~cm}$ after 6 laps (Fig. 7(k)).

\section{CONCLUSION}

The low-cost car-like robot called BioCarBot presented in this paper is able to estimate its velocity and steering angle by means of an Extended Kalman Filer (EKF), using only the OF measurements delivered by two novel downward-facing LMSs. These novel LMSs have the following advantages over those presented, for instance, in [11], [12]:

- the OF measurements are robust to high-dynamic-range lighting conditions (in a 5-decade range with sharp changes of up to 2 decades within $0.5 \mathrm{~s}$ ) and do not depend on the visual patterns encountered;

- the refresh rate of the OF measurements is constant and relatively high $(333 \mathrm{~Hz})$, and does not depend on the bandwidth of the band-pass filter;

- the resolution on the OF measurements is nearly constant and relatively high (up to $0.05 \frac{\mathrm{rad}}{\mathrm{s}}$ ), and can be set at whatever value required;

- the OF measurement range is relatively wide (from 1 to $10 \frac{\mathrm{rad}}{\mathrm{s}}$, i.e. 50 to $550 \frac{\circ}{\mathrm{s}}$ ) and can also be adjusted as required.

Although we focused here only on the information provided by the LMSs, data provided by other sensors (such as wheel encoders, IMUs and GPSs) could be integrated in order to achieve greater robustness and eventually obtain a precise robot's positioning.

Outdoor tests involving various ground and lighting conditions and various trajectories are now under way.

\section{ACKNOWLEDGMENTS}

We are most grateful to M. Boyron and J. Diperi for their involvement in the electronic and mechanical design of the sensors and the robot. We also thank S. Viollet, A. Manecy and F. Colonnier for their helpful suggestions and comments during this study, E. Vanhoutte for the photographs, S. Allano and F. Guillemard for their assistance and J. Blanc for correcting and improving the English manuscript.

\section{REFERENCES}

[1] S. Panzieri, F. Pascucci, and G. Ulivi, "An outdoor navigation system using GPS and inertial platform," IEEE/ASME Trans. Mechatronics, vol. 7, no. 2, pp. 134-142, 2002.

[2] A. Georgiev and P. Allen, "Localisation methods for a mobile robot in urban environments," IEEE Trans. Robot. Autom., vol. 20, no. 5, pp. 851-864, 2004.

[3] S. Chhaniyara, P. Bunnun, L. D. Seneviratne, and K. Althoefer, "Optical Flow Algorithm for Velocity Estimation of Ground Vehicles: A Feasibility Study," Int. J. Smart Sens. Intell. Syst., vol. 1, no. 1, pp. 246-268, 2008.

[4] M. Killpack, T. Deyle, C. Anderson, and C. C. Kemp, "Visual odometry and control for an omnidirectional mobile robot with a downward-facing camera," in Proc. IEEE Int. Conf. Intell. Robot. Syst., 2010, pp. 139-146.

[5] S. Kim and S. Lee, "Robust velocity estimation of an omnidirectional mobile robot using a polygonal array of optical mice," Int. J. Contr. Autom. Syst., vol. 6, no. 5, pp. 713-721, 2008.

[6] R. Ross, J. Devlin, and S. Wang, "Toward refocused optical mouse sensors for outdoor optical flow odometry," IEEE Sensors J., vol. 12, no. 6, pp. 1925-1932, 2012.

[7] I. Nagai, K. Watanabe, K. Nagatani, and K. Yoshida, "Noncontact position estimation device with optical sensor and laser sources for mobile robots traversing slippery terrains," in Proc. IEEE Int. Conf. Intell. Robot. Syst., 2010, pp. 3422-3427.

[8] D.-H. Yi, T.-J. Lee, and D.-I. Cho, "Afocal Optical Flow Sensor for Reducing Vertical Height Sensitivity in Indoor Robot Localization and Navigation," Sensors, vol. 15, no. 5, pp. 11 208-11 221, 2015.

[9] F. Ruffier, S. Viollet, S. Amic, and N. Franceschini, "Bio-inspired optical flow circuits for the visual guidance of micro air vehicles," in Proc. IEEE Int. Symp. Circuits Syst., 2003, pp. 846-849.

[10] F. Expert, S. Viollet, and F. Ruffier, "Outdoor field performances of insect-based visual motion sensors," J. Field Robot., vol. 28, no. 4, pp. 529-541, 2011.

[11] G. Sabiron, P. Chavent, T. Raharijaona, P. Fabiani, , and F. Ruffier, "Low-speed optic-flow sensor onboard an unmanned helicopter flying outside over fields," in Proc. IEEE Int. Conf. Robot. Autom., 2013, pp. 1742-1749.

[12] F. Expert and F. Ruffier, "Flying over uneven moving terrain based on optic-flow cues without any need for reference frames or accelerometers," Bioinspiration \& Biomimetics, vol. 10, no. 2, p. 026003, 2015.

[13] N. Franceschini, A. Riehle, and A. Nestour, Facets of vision, 1989, ch. Directionally selective motion detection by insect neurons, pp. 360390.

[14] W. Reichardt, Sensory Communication. MIT Press, 1961, ch. Autocorrelation, a principle for the evaluation of sensory information by the central nervous system, pp. 303-317.

[15] S. Mafrica, S. Godiot, M. Menouni, M. Boyron, F. Expert, R. Juston, N. Marchand, F. Ruffier, and S. Viollet, "A bio-inspired analog silicon retina with michaelis-menten auto-adaptive pixels sensitive to small and large changes in light," Opt. Express, vol. 23, no. 5, pp. 5614$5635,2015$.

[16] M. F. Land, "Visual acuity in insects." Annual review of entomology, vol. 42, no. 46, pp. 147-177, 1997.

[17] A. Manecy, N. Marchand, F. Ruffier, and S. Viollet, "X4-MaG: A LowCost Open-Source Micro-Quadrotor and its Linux-Based Controller," Int. J. Micro Air Veh., vol. 7, no. 2, 2015.

[18] A. De Luca, G. Oriolo, and C. Samson, Feedback control of a nonholonomic car-like robot, 1998. 